\title{
Cardiologia 1963,43.129-132
}

.

\section{Buchbesprechungen - Book Reviews - Livres Nouveaux}

Rudolf Bauer und Claus Albers: Verhandlungen der Dcutschen Gesellschaft für Kreislaufforschung. 28. Tagung, Bad Nauheim, 27.-29. April 1962. Thema: Essentielle Hypertonie. Dr. Dietrich Steinkopff Verlag, Darmstadt 1963. XLV + 480 S., 163 Abb., 48 Tab. DM 70.-.

Die Nauheimer Tagung von 1962 war in der Hauptsache der «essentiellen Hypertonie» gewidmet. In Hauptreferaten wurden die Pathogenese (Peart: Hu-morale Faktoren; Gross: Hormonale Faktoren; Hoitz: Brenzkatechinamine; Mertz: Wasser- und Mineralhaushalt), die Klinik (Wollhcim: Nosologie; Mueller: Epidemiologie; Heintz: Klinik und Differentialdiagnose; Reubi: Beteiligung der Niere; Spang: Kardiale Komplikationen; Bernsmaier: Hirnkreislauf) und die Therapie (Bechgaard: Spontanverlauf; Kroneberg: Pharmakologie der Antihypertensiva; Losse: Therapie der benignen Hypertonie; Arnold: Therapie der malignen Hypertonie) entsprechend dem heutigen Forschungsstande abge-handelt. Sowohl in den Hauptreferaten als auch in mehreren Einzelmitteilun-gen wurden besondere Probleme wie die reno-vaskuläre Hypertonie (Nieren-arterienstenosen) und die Decarboxylasehemmung (Alpha-methyl-dopa) als neues therapeutisches biochemisches Prinzip eingehend dargestellt. Ferner be-fassen sich eine große Zahl von interessanten Kurzreferaten mit weiteren Pro-blemen der Hypertonie und der klinischen Kardiologie und Angiologie.

Das Buch orientiert den am Problemkreis der essentiellen Hypertonie so wohl klinisch als auch pathophysiologisch oder experimentell interessierten Arzt in übersichtlicher Weise und aus erster Hand liber den heutigen Stand der Erkenntnisse. Der Referent hat nur einen Vorbehalt anzubringen, der auch für viele andere Tagungsberichte gilt und der die lange Verzögerung (ca. 1 Jahr) zwischen Kongreßdatum und Veröffentlichung betrifft. Dadurch verlieren Tagungsberichte und Kongreßreferate bestimmt an Aktualität und kompromittieren eventuell auch Prioritätsrechte. F. Schaub, Zurich.

LipopГoteidlipasc. Symposium vom 1. und 2. Juni 1962 an der 2. Medizin. Uni-versitätsklinik Wien. Herausgegeben von H. Braunsteiner, S. Sailer und F. Sandhofer. Bibl. Haemat. Fasc. 15. S. Karger, Basel/New York 1963. IV + 148 S., 58 Abb., 4 Tab. sFr. 27.-.

Die Lipoproteidlipase ist das bei der Klärwirkung durch Heparin beteiligte Enzym. Die vielfach noch ungelöste und zweifellos von manchen Klinikern in ihrer Bedeutung überschätzte und therapeutisch zu früh übernommene Klärwirkung des Heparins wird eingehend und kritisch besprochen. Die Bestim-mungsmethodik, die Wirkungsweise und die physiologische und klinische Bedeutung des Lipoproteidlipase-Systems sowie die Beziehungen zwischen Blut-fetten einerseits und Gerinnung und Atherosklerose andererseits erfahren durch die im deutschen Sprachgebiet in diesem Forschungsgebiet führenden Autoren (Schettler, Zöllner, Braunsteiner, Hartmann usw.) eine klare, den heutigen un-vollständigen Kenntnissen angepaßte zurückhaltende Analyse und klare Dar-stellung. Die Monographie wird ohne Zweifel Ärzten, die sich für die Klär-

130 Buchbesprechungen - Book Reviews - Livres Nouveaux 
wirkung des Heparins und ihre Beziehung zur Atherosklerose interessieren, als eilie wertvolle übersicht über den heutigen Wissensstand dieses Problemkreises dienen.F. Schuub, Zurich.

G. E. Burch and N. P. De Pusquale: Hot Climates, Man and His Heart. C. G. Thomas, Springfield 1962.

Die Resultate persönlicher Untersuchungen über die Wärmeregulierung des Organismus, die von den Autoren Jahre hindurch mit eigenen Methoden betrieben wurden, werden ausführlich berichtet. Es wird besonders unterstrichen, daß die kutane Gefäßerweiterung, die nötig ist, um einen adäquaten Wärmeverlust durch die Haut hindurch zu gewährleisten, eine beträchtliche Zunahme der Herzbelastung erfordert. Beim Übergang von einer Umgebung mit gemäßigtem Klima in ein feucht-warmes Milieu kann sich die Herz belastung bei gesunden Personen um das 4 fache steigern. Die unausgeglichenen Herzkranken sind nicht in der Lage, diese Erhöhung der Belastung zu ertragen, und haben eine ernsthafte Verschlechterung ihres Zustandes zu erwarten. Das von den Autoren vorgelegte Bildmaterial ist reichhaltig. Das Werk ist durch die Ausführlichkeit und Gründlichkeit, mit der das Thema behandelt wird, wie auch durch die Originalität der durchgeführten Untersuchungen von beachtlichem Wert. A. Selvini, Milano.

Th. Mottu: Le cathétérisme ct l'angiocardiographie des cavités eardiaques gauches et de Гaorte par la méthode artérielle retrograde. Ed. Médecine et Hygiene, 1962.75 p., 54 planches. 25 frs. Quel plaisir de lire une monographie aussi bien faite. On entre tout de suite dans le vif du sujet par un historique finement nuance des méthodes modernes sanglantes d'exploration des cavités gauches. L'auteur a pris un soin minutieux à nous retracer la série des aventures que représentent ces investigations, parfois risquées, mais devenues indispensables avec les progrès de la cardiochirurgie. II faut le louer particulièrement d'avoir rendu hommage aux travaux d'autrui et le louer aussi de son esprit critique et de sa bienveillance de gentleman.

II plaide avec conviction le cathétérisme artériel retrograde en s'appuyant sur des recherches solidement documentées.

Cette monographie fait honneur à $\Gamma$ Ecole Duchosal de Geneve. Elle vient à son heure... à la dernière heure peut-être ? - La ponction ventriculaire gauche, tellement plus expéditive, plus élégante, ne va-t-elle pas détrôner le cathétérisme artériel retrograde ? L'auteur pose lui-même la question ce qui montre qu'il est objectif et ne se laisse pas aveugler par sa conviction du moment. II sait bien que la conviction n'est pas un argument, d'autant plus que depuis lors ГEcole Duchosal plaide aussi, non sans pertinence, le cathétérisme des cavités gauches par la voie transseptale droite.

La documentation personnelle et Ticonographie sont presentees sous forme de planches bien composées. Les illustrations auraient gagné à bénéficier d'une impression plus luxueuse. C'est la seule restriction que $\Gamma$ on peut formuler.

J'estime qu'un livre aussi bien compose dépasse le cercle des seuls cardio-logues et qu'il sera profitable à tous les médecins. Ivan Mahaim, Lausanne.

Buchbesprechungen - · Book Reviews - Livres Nouveaux 131

M. J. Halhuber und H. Kirchmciir: Notfälle in dor iiineren Medizin. Sechste, neubearbeitete und erweiterte Auflage. Urban \& Schwarzenberg, Münehen und Berlin 1963. XIII + 414 S., 14 Abb. DM 21.-. 
Das heute 6 Jahre nach seinem ersten Erscheinen in sechster Auflage vor-liegende Buch beschäftigt sich in einem ersten Abschnitt mit alarmierenden Symptomen, wobei vor allem die Bewußtlosigkeit, Atemnot, Anurie, der Herz-schmerz und der Bauchschmerz eingehend besprochen werden. In einem zwei-ten Abschnitt werden diejenigen akut bedrohlichen Zustandsbilder besprochen, die bei differentialdiagnostischen internmedizinischen Überlegungen immer wieder in Betracht gezogen werden müssen. Das Buch entspricht einem Be-dürfnis des praktischen Arztes und des Internisten, die hier bei selteneren Zuständen notwendige Hinweise finden und bei geläufigen Krankheitszustän-den die getroffenen Maßnahmen überprüfen können. W. Siegenthaler, Zurich.

Moi·bidität an koronaren peripheren und cerebralen Arterienverschlüssen.

Bericht über die 1. Jahresversammlung der Schweizerischen Gesellschaft für Angiologie mit Ansprache des Präsidenten R. Nissen, Basel, 25. Mai 1962. Herausgeber: L. K. Widmer, Basel, und J.-L. Schelling, Lausanne. Bibliotheca Cardiologica, Fasc. 13. S. Karger, Basel/New York 1963. 152 p, 64 fig., 22 tab. sFr./DM 32.-.

Der Band bringt in extenso die Hauptreferate und die Voten der aufgefor-derten

Diskussionsredner der 1.Tagung der Schweizerischen Gesellschaft für Angiologie. Th. R.

Dawber referierte über die aus der bekannten «Framingham Study» evident gewordenen aetiologischen und epidemiologischen Faktoren der koronaren Herzkrankheit. Ohne Zweifel stellt die «Framingham Study» die bis heute wissenschaftlich einwandfreieste und aufschlußreichste Analyse der Epi-demiologie und Aetiologie des Herzínfarkts dar. L. McDonald besprach die um-strittene Rolle der Thrombophilie in der Genese des Infarktes. In der Diskussion kamen vor allem der atherogene Einfluß des Diabetes (inkl. des latenten Diabetes), der Blutfette und anderer biochemischer Störungen sowie der Hyper-tonie zur Sprache. L. K. Widmer berichtete über die Morbidität an Gliedmaßen-arterien-Verschluß bei 6400 Berufstätigen. In der Diskussion wurden die relative Häufigkeit von Gliedmaßenverschlüssen in der zweiten Hälfte des 4. Jahr-zehnts, die diagnostische Bedeutung der Auskultation von Aorta und Glied-maßenarterien, die Rolle der Zusammensetzung des Kollektivs für vergleichende statistische Studien und die Tatsache, daß Gliedmaßenverschlüsse mehrheitlich Symptome einer diffusen Arterienerkrankung sind, besonders hervorgehoben. E. C. Hutchinson und E. Strieker behandelten die Pathologie der extracranialen Arterien der cerebralen Zirkulation bzw. der cerebralen Arterien. In der Diskussion wurden vor allem die zeitlichen und zahlenmäßigen Relationen der Hemiplegie zum Herzinfarkt und Gliedmaßenarterienverschluß (etwa 10 bis 15 Jahre später und etwa 5-1Omal seltener als der koronare oder periphere Arterienverschluß), die Pathogenese der Hemiplegie ( $5 / 8$ der Erweichungen zeigen weder Stenosen noch Verschlüsse; Bedeutung von Blutdruckabfall, Ar-rhythmien, Spasmen, Oedem, venöser Stase usw.) und die Früherfassung cere-

132 Buchbesprechungen - Book Reviews - Livres Nouveaux

braler Durchblutungsstörungen durch Berücksichtigung transitorischer İschä-miezeichen betont. Das Buch kann jedem Arzt empfohlen werden als moderne Informationsquelle der heutigen Erkenntnisse über die Arterienverschlüsse in verschiedenen Organen. F. Schaub, Zurich.

A. Moll und F. Hamacher: Der Herzinfarkt im jüngeren Lcbensalter. Bei-träge zur praktischen Medizin, Heft 44. Verlag Ferdinand Enke, Stuttgart 1962. 52 S., 8 Abb.

Die an der Inneren Abteilung des Stadtkrankenhauses Rüsselsheim/Main tätigen Autoren haben ihre eigene Serie von Myokardinfarkt bei Menschen unter 40 Jahren durch Umfrage bei 24 Kliniken bzw. Ärzten auf insgesamt 
67 Fälle erweitert. Sie berichten nun in einer kurzgefaßten, in gutem Stil geschriebenen Monographie über diese Arbeit. Ihr wesentliches Ergebnis ist, daß der Myokardinfarkt «Jugendlicher» keine Krankheit sui generis zu sein scheint, sondern zur «gewöhnlichen» koronaren Herzkrankheit gehört. Es waren im besonderen keine Befunde zu erheben, welche an eine aetiologische Bedeutung entzündlicher Prozesse denken lassen (7 Autopsien). Die Besonderheiten der Serie (Myokardinfarkt meist 1. Manifestation der Krankheit; seltener Koexistenz von Hypertension, Diabetes mellitus oder übergewicht; körperlicher Aktivität der Betroffenen größer; bessere Prognose) erklären sich wohl zum Teil allein durch das niedrigere Durchschnittsalter der Patienten. - Die Publikation stellt einen wertvollen Beitrag zur Kenntnis der koronaren Herz krankheit dar. W. Schweizer, Basel.

H. P. Koepchen: Die Blutdruckrhythmik. Eine Untersuchung über die Bedeutung der zentralen Rhythmik für die ncrvöse Krcislaufsteuerung.

D. Steinkopff-Verlag, Darmstadt 1962. X + 139 S., 48 Abb., 3 Tab. DM 36.-. Die aus dem Physiologischen Institut der Universität Göttingen stammende Monographie behandelt die gelegentlich unter dem vagen Begriff der «Blutdruckwellen» segelnden und allgemein etwas vernachlässigten Vorgänge, welche in spontanen, rhythmischen Schwankungen des Blutdruckes bestehen. Es ist das Verdienst des kritischen Autors, diese «Blutdruckrhythmik» einer eingehenden physiologischen Analyse unterzogen und sich begrifflich, theoretisch und experimentell intensiv mit diesem Phänomen auseinandergesetzt zu haben. Die Blutdruckrhythmik wird auf rhythmisch tätige Substrate der medulla oblongata zurückgeführt und zu anderen vegetativen Zentren, die ebenfalls rhythmisch agieren (vor allem Atemzentrum), in Beziehung gesetzt bzw. abgegrenzt. Der Einfluß peripherer Afferenzen und die Prinzipien der Kreislaufsteuerung werden kritisch und genau dargestellt. Physiologen und Kliniker, die an der Grundlagenforschung der Blutdruckregulation und an den Problemen der zentralen Rhythmik interessiert sind, werden im Buch von Koepchen einen reichen Quell vieler Anregungen und experimenteller Daten finden und die zuverlässige Darstellung der mit der Blutdruckrhythmik zusammenhängenden Fragen mit Gewinn benützen. F. Schaub, Zurich. 